\title{
Applying a Bauhaus design approach to conceptualize an integrated system of mental health care: Lessons from a large urban hospital
}

\author{
Thomas Ungar $^{\mathrm{ab} \star}$, Marlene Taube-Schiff ${ }^{\mathrm{b} \star}$, and Vicky Stergiopoulos ${ }^{\mathrm{bc} \dagger}$ \\ ${ }^{a}$ North York General Hospital, Mental Health Program, 4001 Leslie Street, 8N8 12C, North York, \\ Toronto, ON M2K 1E1, Canada; ' ${ }^{\circ}$ Department of Psychiatry, University of Toronto, 250 College \\ Street, Toronto, ON M5T 1R8, Canada; 'St. Michael's Hospital, 30 Bond Street, Ste. 17-038, Toronto, \\ ON M5B 1W8, Canada \\ *thomas.ungar@nygh.on.ca, marlene.taube-schiff@nygh.on.ca \\ ${ }^{\dagger}$ Present address: Centre for Addiction and Mental Health, 100 Stokes Street, Toronto, ON M6J 1H4, \\ Canada.
}

OPEN ACCESS

Citation: Ungar T, Taube-Schiff M, and Stergiopoulos V. 2016. Applying a Bauhaus design approach to conceptualize an integrated system of mental health care: Lessons from a large urban hospital. FACETS I: 173-186. doi:10.1139/ facets-2016-0016

Editor: Paul Greenman

Received: April 21, 2016

Accepted: July 22, 2016

Published: November 2, 2016

Corrected: November 22, 2016

Copyright: (C) 2016 Ungar et al. This work is licensed under a Creative Commons Attribution 4.0 International License (CC BY 4.0), which permits unrestricted use, distribution, and reproduction in any medium, provided the original author(s) and source are credited.

Published by: Canadian Science Publishing

\begin{abstract}
We have applied a Bauhaus design lens to inform a visual conceptual framework for a rational mental health care system. We believe that Canada's healthcare system can often be fragmented and does not always allow for service delivery to easily meet patient care needs. Within our proposed framework, the form of services provided follows patient- and healthcare-centred needs. The framework is also informed by the ethics and values of social responsibility, population health, and principles of quality of care. We review evidence for this framework (based on need, acuity, risk, service intensity, and provider level) and describe patient care pathways from intake/triage to three patient-centred tiers of care: (1) primary care (low needs), (2) acute ambulatory transitional care (moderate needs), and (3) acute hospital and complex care (high needs). Within each tier, various models of care are organized from low to high service intensity as informed by reports from the British Columbia Ministry of Health and the World Health Organization. We hope that our model may help to better conceptualize and organize our mental health care system and help providers clarify roles, responsibilities, and accountabilities to improve quality of care.
\end{abstract}

Key words: mental health, healthcare system, integrated, healthcare delivery

\section{Introduction}

The mental health care system is fragmented, and patients are often unable to access clear entry points or move through the system in a direct and beneficial manner (Government of British Columbia-Ministry of Health 2012). North York General Hospital (NYGH) is an acute care general hospital, providing various mental health services to adult, geriatric, child, and adolescent populations. Our wide-ranging demands result in challenges to understanding our mandate and adhering to best practices. We believe that other acute care general hospitals and mental health programs struggle with similar issues. Increasing demand and service volumes and stagnant or shrinking resources have resulted in lengthening wait times for outpatient consultations and difficulty ensuring timely follow-up. We have designed a visual conceptual framework for a rational mental health care system. We hope this can aid organizations, policy planners, 
and clinicians to clarify responsibilities and professional roles as well as enable the provision of best practices. We have applied elements of the philosophy of Bauhaus design such that the form of our service framework follows patient-centred and healthcare system needs (Grumbach 2009). It also provides an ethical and value-based structure for the assignment of responsibilities, resources, models of care, and accountabilities. Our framework has been informed by a review of the literature as well as by the experiences of author T. Ungar as a family physician and, currently, as a psychiatrist, clinical chief, and hospital physician-in-chief within an acute care general hospital with ambulatory healthcare services. We would like to specifically highlight a recent report on collaborative care models from the Ministry of Health in British Columbia (Government of British Columbia-Ministry of Health 2012) as well as a World Health Organization (WHO) publication on optimal services for mental health (World Health Organization (WHO) 2003). Elements of these models have informed our framework.

We have put considerable thought into the decision not to use geography or location of care as the primary organizing principle for health services or funding (e.g., hospital vs. community care). We believe that this organizing principle limits health service design. Instead, we use clinical functionality as the organizing principle including intensity of service, patient-centred needs, and required healthcare provision enablers.

Understandably, a natural tension may exist between a rational Bauhaus design of health care and political pressures. Mental health services have been chronically underfunded in many jurisdictions, including Canada. For example, it has been cited that over 6.7 million Canadians (18.1\% of the population) experience mental health issues (Lurie 2014). The direct and indirect costs to the Canadian economy have been estimated at $\$ 50$ billion per year with a projection of collective costs increasing to $\$ 2.5$ trillion over the next 30 years (Lurie 2014). Within a government-managed public mental health care system, political realities and sensitivity to voters within specific geographic regions may contribute to decisions about health resource allocation, such as service funding and location. We hope the logical and rational "form follows function" framework helps decision makers to ensure that ethical and principled decisions override temporary political influences.

\section{Current organization of mental health care delivery}

In the province of Ontario, and indeed the rest of Canada, the current mental health care system is primarily organized from a structural/institutional perspective. Institutions, such as hospitals, often play a central role in determining patient needs, prioritizing areas of focus (often driven by budget pressures), and designing facilities. Thus, a hospital's system does not always prioritize patient needs, but rather the needs of the organization. We invite the reader to imagine this as analogous to an elaborate edifice of a building that does not serve the purpose of its users. Thus, the hospital "edifice" is not always practical or rational. For example, there is often a harsh divide between inpatient services and discharge to ambulatory or community resources. In an attempt to respond to these dominant structural and institutional barriers, initiatives such as Health Links (Ontario Ministry of Health and Long-Term Care 2016a) and patient navigators have been created. Similarly, current funding models may be barriers or disincentives to best care, as traditional budgets are organized first around institutions such as hospitals, and only subsequently in the service of clinical patient needs. At times, the budgetary interests of the institution and that of patient needs may be in conflict.

Another principle of the current organization, beyond the institution, is the edifice of server provider groups. Both professional and healthcare provider groups have needs and interests that prioritize their role within the healthcare system beyond patient needs. For example, physicians may not want to provide services after hours, or to the most acutely ill, and may resist changes or threats to their professional autonomy. Thus, this principle of the current organization places institutions at the centre, providers second, with patient needs and functionality often being served last. 
In contrast, patient- and family-centred care initiatives are similar to a Bauhaus design of the delivery of health care in that they place the patient at the centre of the values and decision making of the hospital. The movement towards integrated care collaboratives (Government of British ColumbiaMinistry of Health 2012; Porter and Lee 2013) as well as accountable care organizations (Centers for Medicare \& Medicaid Services 2015) is an attempt to respond to and overcome the budgetary and financial barriers to providing adequate patient-centred needs. Katon and Unützer (2011, p. 308) describe the "triple aim" of collaborative care approaches; see also Berwick et al. (2008). This includes enhancing access to evidence-based practice, greater provider and patient satisfaction, enhanced health outcomes, and decreased costs for health care (Katon and Unützer 2011). We believe that these aims have been illustrated in our framework as well.

\section{Bauhaus design in the delivery of health care}

The Bauhaus school of design was initially founded in 1919 by Walter Gropius (Droste 2006), who envisioned a philosophy of "form follows function". Within this movement was the importance of rational and simplified design. A complete review of the history and development of Bauhaus design would extend far beyond the reach of this paper. However, a discussion of the philosophy can demonstrate relevance to the healthcare system (Grumbach 2009). If we can extend the idea that a chair's structure should logically flow from the function of its proposed use, we could envision how a primary care practice should create initiatives to improve the accessibility of services, such as advanced scheduling systems and increased clinical hours (Murray et al. 2003). Within our framework, we have envisioned the needs of patients as they flow through the mental health care system from a clear point of triage entry along pathways of services that meet their functional needs-both not being given more services than what would be adequate, and not being given less. We believe that this philosophy embraces the spirit of "form follows function".

Grumbach (2009) also described the principles of this system, which involve: (1) healthcare workers operating at the top level of their skill set and with others in collaborative teamwork efforts, which requires an understanding of professional roles by all team members; (2) healthcare systems delivering care matching the epidemiological profile of patients served; (3) the physical design of the healthcare system allowing for improved patient flow and clinical care; (4) resources following need; and (5) the involvement of patients in designing the healthcare system. We will demonstrate some of these principles within our conceptual framework.

\section{Intake/triage process}

As illustrated by our framework (see Fig. 1), individuals ideally access care through a single point of entry; or at least as few as necessary. Within our general hospital we have recognized challenges resulting from multiple points of entry and intake to our internal services. We believe that the analogy of an "air traffic control office" captures our goal for an intake and triage system, rather than multiple individual runways of arrivals and departures to our services. One existing approach for triage used in emergency rooms is the Canadian Triage and Acuity Scale (Bullard et al. 2014) and Canadian Emergency Department Information Systems (CEDIS) identifiers for mental health. In addition, some practitioners are also using the level of care utilization system (LOCUS) tool (American Association of Community Psychiatrists 2010). We believe that this may be a useful starting point upon which to build our broader system intake/triage point of entry (Canadian Association of Emergency Physicians (CAEP) 2016). The LOCUS assesment tool is currently used for this purpose in some organizations and regions. If patients enter the system in a clear and directed way, the chances of appropriate services following their needs are much more likely than with an unsystematic or disorganized approach. 

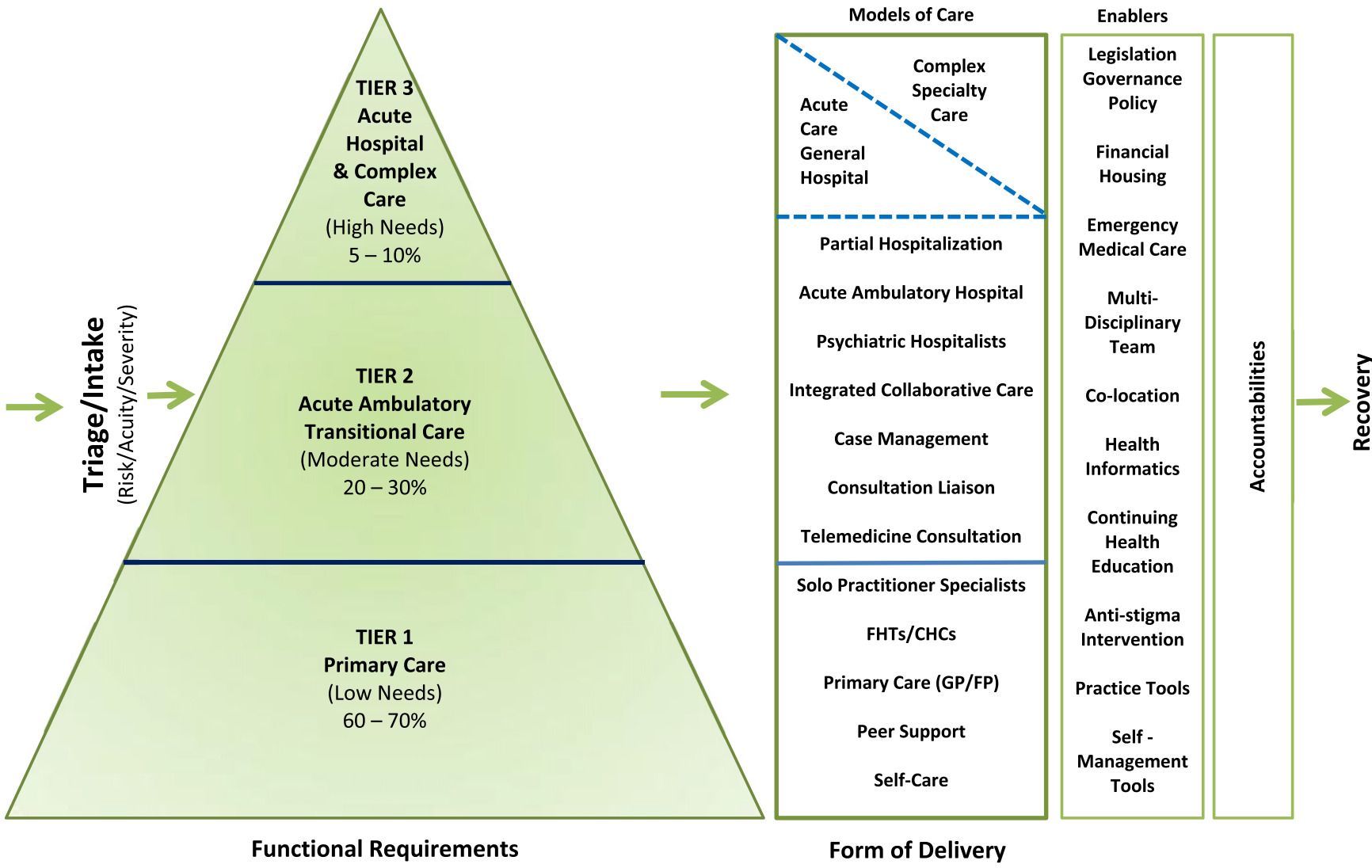

Fig. 1. Bauhaus mental health system model (concept and figure created by Dr. Thomas Ungar prior to the drafting of this manuscript). This figure illustrates how the form of services within the mental health care system follows patient needs. Patient care pathways begin at intake/triage and flow through to three patient-centred tiers of care: (1) primary care (low needs), (2) acute ambulatory transitional care (moderate needs), and (3) acute hospital and complex care (high needs). Within each tier, various models of care are organized from low to high service intensity. All models of care require enablers to function optimally and must meet certain accountabilities, as further detailed within our paper. FHT, family health team; CHC, community health centres; GP, general practitioner; FP, family physician.

\section{Services of care pyramid}

Our services of care pyramid was adapted from the model of care pyramid that originated to guide treatment for complex diabetes care (York Teaching Hospital 2016) and from a model produced by the WHO (2003). We describe each level of service based on the functional clinical needs of patient care, as informed by the following: acuity, risk, illness severity, service intensity, and required service provider skill. We hope that these conceptual bundles allow for planning patient care across the functional continuum of needs. Moving to the "form" or care required to meet these functions, we have included a variety of services and models of care. We do not believe that there is a "single bullet" to appropriately meet differing care needs and recognize that we have excluded some models of care and services within our framework. However, the intention of our model is a purposeful design to embrace simplicity in order to be useful.

As depicted in Fig. 1, we have ascribed a percentage of population mental health needs that are managed within each tier of the services pyramid. 


\section{Tier l: low needs-self and primary care}

The bottom tier of the pyramid illustrates the high percentage of mental health issues and needs that are cared for with lower intensity services. Models of care for this tier include self-care and self-management. Peer support is also an emerging model of care and is available in some regions.

Within Canada, a large proportion of mental health issues are treated within a primary care setting. In fact, it has been found that over $50 \%$ of Canadians use healthcare services with their primary care physician for issues related to mental health (Kates et al. 2016). The WHO recently released a report entitled "Integrating mental health into primary care: A global perspective" (Greenhalgh 2009), which highlighted reasons for mental health being treated in primary care facilities. These include frequent co-occurrence of mental and physical difficulties; increased availability of access to primary care for mental health; and primary care being less expensive than specialized care within a hospital system. For most people, a primary care physician is their first point of contact for health care. Strikingly, it has been found that between $20 \%$ and $76 \%$ of patients who commit suicide had visited their primary care physician the month before (Luoma et al. 2002; Carrigan and Lynch 2003).

Loeb et al. (2012) investigated the viewpoints of primary care physicians regarding patient, physician, and system factors impacting care for patients presenting with mental health and medical issues. Physicians indicated that the type of mental illness, acuity of presentation, and patient communication style are all important issues. Primary care physicians also wanted enhanced training regarding mental health issues and to take part in collaborative care models (Loeb et al. 2012). Patients described frustration regarding issues such as inadequate resources and disjointed care. Given the high incidence of mental illness within primary care, this study provides a compelling narrative of areas for improvement to enable enhanced provision of mental health services within primary care settings.

Different primary care models have been well established, including the traditional roles of general practitioners and family physicians (i.e., primary care physicians in the USA), and paediatricians for children and adolescents. In 2005, 184 Family Health Teams (FHTs) were created in Ontario to broaden family healthcare delivery (Ontario Ministry of Health and Long-Term Care 2016b). As described by the Ontario Ministry of Health and Long-Term Care (2016b), FHTs include family physicians, nurse practitioners, registered nurses, social workers, dietitians, and occasionally other specialized professionals. Currently, over three million Ontarians receive care through FHTs in 200 communities (Ontario Ministry of Health and Long-Term Care 2016b). Integrating mental health within primary care also de-stigmatizes mental illness by preventing the segregation that may emerge from treatment in a standalone mental hospital model (Falk and Allebeck 2002; WHO 2003). Thus, FHT models play an important role within a Bauhaus model of health care, as patient needs can be met along the continuum of services that are provided.

We have also listed specialists in the primary care tier based on their clinical functioning and activity rather than on their level of training. Specialists fulfilling mid- or highest-needs clinical roles are listed again in tier 2 or 3, although we use the term "consultant" or "hospitalist" to distinguish their level of functionality within those tiers. Solo practitioner (i.e., often community office-based) specialists, such as psychiatrists, private practice psychologists, and other allied health therapists treat individuals presenting with a variety of mental health issues that are often lower acuity, currently stable, or optimized. Many solo practitioner psychiatrists are providing psychotherapy and low intensity services to a fairly stable, less acute patient population. In this regard, despite training and certification as medical specialists, they are meeting patient clinical needs at the lower end of their initial clinical training potential and functioning more in a primary mental health care capacity. We recognize that this statement is likely controversial and possibly upsetting to the profession. But, based on the 
principles of Bauhaus design, this reflects the current clinical function of these physicians relative to the broader health system and population. Within a Bauhaus-designed healthcare system, specialists would be working at the top level of their skill set (Grumbach 2009). This would allow for better matching of the needs of patients to services available.

\section{E-Health enabler}

We believe that there is tremendous opportunity for transformative disruption within tier 1 as a result of the flurry of self-management apps; for a recent review, see Bakker et al. (2016). Some examples of these apps include: CBT-i Coach http://t2health.dcoe.mil/apps/CBT-i (Kuhn et al. 2016), Moodkit http:// www.thriveport.com/products/moodkit/, DBT Diary Card and Skills Coach http://www.diarycard.net/, and CBT Bariatric https://cbtbariatric.com/ (Zhang et al. 2015). Public health information and internet-based self-care for the public are also sources of self-management. One caveat is that there is a lack of standardization of this information, and, therefore, the public can be at risk of being bombarded with information that lacks both standardization and accuracy. To support professional development, web-based accredited health education is growing. Clinical tools and practice enablers for primary care providers are increasingly being embedded at point of use, such as the Patient Health Questionnaire (PHQ-9; Spitzer et al. 1999) in primary care electronic health records, as well as clinical decision support for prescribing medications within electronic charts. For example, alerts and warnings are often available within these systems to support quality of care and population-based management.

\section{Tier 2: moderate needs-acute ambulatory transitional care}

Transitional care services can bridge between the highest-level involvement of acute hospital emergency, inpatient and complex specialized care for severe mental illness (SMI), and lower needs services (e.g., primary care). Tier 2 models include acute ambulatory programs as well as partial hospitalization programs (Granello et al. 1999; Yanos et al. 2009; Thatte et al. 2013; Association for Ambulatory Behavioral Healthcare 2014; Lenz et al. 2014). Partial hospitalization programs were created in the 1930s and, since that time, have conventionally been viewed as an alternative to inpatient hospitalization for individuals presenting with acute psychiatric symptoms not requiring overnight stay or 24-hour safety monitoring (Marshall 2003). These services are very much in keeping with the Bauhaus philosophy of patient needs being matched by appropriate care.

Typically, transitional care services are delivered to a more acute patient population than individuals treated within our lower models of service tier. At NYGH, we have used the name Acute Ambulatory Integrated Transitional Care to describe our tier 2 moderate needs models of care. When conceptualizing this tier of service, we moved from the dominant (but limited) binomial planning conceptual model (i.e., hospital vs. community) to a three-tier model. These services were developed as a response to the need to articulate the function of outpatient mental health services within an acute general hospital and differentiate them from the lower level tier 1. Central health planners believed that only inpatient care should be provided by a hospital with other services divested and provided in "the community." These types of transitional services are geared towards patient needs that are no longer emergent or most acute, but sub-acute or post-acute, as the individual has not fully stabilized. These services exceed those provided in primary care settings.

The services aggregated and co-located within NYGH include the following: telemedicine consultation (Hilty et al. 2013); collaborative shared care (Kates et al. 2011); reversed shared care (Ungar et al. 2013); outpatient psychiatry; Partial Hospitalization Day Programs; mental health and justice treatment and support services; and community legal aid services and addiction program for adults and transitional aged youth. Low-intensity case management for individuals receiving these services is also depicted in tier 2 of our model (see Fig. 1). 
Although our move from a binomial to a trinomial model might be interpreted as being conceptualized from an institutional perspective, we still believe that it holds true to the philosophy of Bauhaus design. Given that patient needs were not being met in our organization (where options were either inpatient services or community resources), a service needed to be created that better addressed patient needs (i.e., form follows function). Many individuals with psychiatric issues require something that is "in between" higher and lower acuity. This type of model can be applied within other acute ambulatory hospitals as well, taking into account the patient profile and overarching needs.

As stated earlier, we acknowledge the role of psychiatrists as medical specialists. However, to better differentiate their functional roles within the healthcare system, we use a novel term "psychiatric hospitalist" (Society of Hospital Medicine 2016) to describe those who provide care to patients with more complex and acute care needs, such as those seen within tier 2 ambulatory outpatient care, consultation-liaison services, partial hospitalization, and (or) tier 3 inpatient care. Psychiatrists functioning as hospitalists in this mid-tier often perform multiple clinical roles. These practitioners often have privileges at a hospital and have on-call duties, allowing them to more seamlessly meet patient care needs, such as facilitating inpatient admission. This is all in keeping with the continuum of services required by patients presenting with more acute conditions.

\section{E-Health enabler}

An enabler of tier 2 is telehealth consultation between patients and families and primary care and other providers. Emails and texting can also help to facilitate consultation communication. For example, the Ontario Telemedicine Network has set up a secure network to facilitate telemedicine services between patients and providers. Telemedicine team-based video conferencing can also help to enable multidisciplinary collaboration and capacity building.

\section{Tier 3: high needs-acute inpatient/complex/severe/long term}

The top of our pyramid reflects services devoted to patients with the highest acuity and intensity of needs. As illustrated in Fig. 1, we believe that both acute care general hospitals (both "community" hospitals and academic health science centres) and specialty/tertiary care hospitals are providing care within this peak tier, with the latter assuming a greater role with SMI and residential care needs. One such model of care within this tier is assertive community treatment (ACT) teams. These teams can be part of the hospital but provide high-intensity specialized services to patients with long-term needs in the community (including home visits) (Ziguras and Stuart 2000; Falk and Allebeck 2002; Nelson et al. 2007; Franx et al. 2008; Canadian Mental Health Association Toronto 2015) given that this is where the need resides. Other models and service care delivery in this tier include psychiatric emergency room services, inpatient services, medically supported substance detoxification, and long-term care and support for SMI.

We invite the reader to reflect on how our Bauhaus approach may differ from the current organization of mental health services. For example, general hospitals are often categorized as "secondary" care and specialty mental hospitals as "tertiary" or "quaternary" care. Yet, functionally, we believe that they are both providing services of high acuity, intensity, and risk. Given that the services delivered within these types of institutions can sometimes blur, we have depicted a broken diagonal line in the model of services delivered to patients functioning in the high complex tier (Fig. 1). Of course, some services are only offered in specialized tertiary care facilities, such as forensic services or those for individuals with developmental disabilities. Therefore, although both general hospitals and specialized hospitals are providing care to acute high-risk patients, specialized hospitals and services are likely targeting a different degree of complexity. Patients that are highly acute presenting to a general hospital may at times be equally or more resource demanding than patients at specialized tertiary care services. 
Thus, both general hospitals and specialized services participate and are likely working at the top of their skill set when treating patients in tier 3-another illustration of a Bauhaus principle.

\section{E-Health enabler}

Implementation of electronic health records aids in communication among staff and among other organizations within this tier. Computerized orders (e.g., evidence-based order sets) can allow for ease of workload. Medication reconciliation can also be accomplished through electronic charting. This information is then passed along at discharge to facilitate ongoing medication management. Finally, a common health record allows for information, test results, imaging, medication use, and so on, to be transferred, enabling better quality of care and decreased burden upon the patient.

Overall, we believe that patients can and should move between tiers in the pyramid of services that best fit their mental health needs, acuity of illness, and level of service provider involvement. This again speaks to the form of service delivery matching the functional needs.

\section{Enablers}

With each tier and for each model of care, enablers must be in place to deliver services. For example, all tiers require skilled professionals, supporting legislation and policy, adequate physical infrastructure, and equitable outcome-based remuneration and funding models. More specifically, for the low-needs models of care, enablers might include self-management tools for patients and families, continuing education, and updated clinical tools. For the moderate transitional needs models of care, enablers might include a multidisciplinary allied ambulatory health team to provide care and the facilities to co-locate these services (Curran et al. 2006), telehealth infrastructure, resources for partial hospitalization programs, and professional and hospital credentialing to support the functioning of health providers. For the high complex needs tier, enablers would include the following: acute general hospital emergency care with adequate patient care areas; acute care psychiatric inpatient beds; medically supported substance detoxification; longer term care inpatient beds and care teams; resources such as housing, finances and vocational rehabilitation; policy and legislation for disability and involuntary care; and high-intensity case management. Health informatics is an important means of enabling widespread access to patient information and collaborative integrated communication within all tiers and has been described within each section.

For each of these forms of service delivery, remuneration models and support for providers should recognize differences in training, skill set, and risk incurred in keeping with the principles of interprofessional education (IPE) in clarifying roles and responsibilities (Curran et al. 2006). Unfortunately, the current physician and health system funding and remuneration models inequitably underresource neuropsychiatric conditions (Patel et al. 2016). Furthermore, current remuneration models within the Ontario Health Insurance Plan (OHIP) fee structure often fail to proportionally recognize differences between tier 1 and tier 3 care provision, and low-needs service providers often earn the same income or very close to that of high-intensity providers as part of a historical fee structure. This might be an area of re-evaluation for future service planners.

\section{Accountabilities}

Within each tier and model of care, we propose the development of provider accountabilities. Our intention is not to negate healthcare provider professional judgement or autonomy. However, professional freedoms are not absolute, and principles of social responsibility within a publicly funded health system with limited resources require culpability. For lower needs providers, such as primary care physicians, this might include initiating a treatment trial for low-complex conditions before referring to mid-tier providers. In addition, primary care providers should resume the ongoing care of a person with 
a mental health condition once adequately stabilized or recovered from moderate- or high-intensity models of care. As described, collaborative care models can help facilitate ongoing consultation, as needed. Specialist physicians providing low-acuity services to a small number of patients in an ongoing long-term manner may need to be challenged given extensive wait lists and burgeoning demands on access to mental health services. Similarly, moderate-tier care providers, such as psychiatric hospitalists, need to ensure timely access to consultation and urgent assessment, and they need to be available for rapid backup and support to primary care providers. In addition, ensuring a timely transition of patients to a lower level of care is also important to avoid patients being seen for an extended period of time within a hospital or unnecessarily under the care of a specialist. Interestingly, the role of primary care versus specialty care in terms of provision and transition of care is clear for most areas of medicine but less clear in mental health. Thus, clarifying interprofessional and interorganizational roles and responsibilities is a best practice of interprofessional education/interprofessional care (IPE/IPC) and effective collaborative care.

Quality-of-care initiatives also, of course, align with accountabilities. The Institute of Medicine's (IOM) six principles of quality of care can guide the development of accountabilities for providers and health organizations (Institute of Medicine Committee on Quality of Health Care in America 2001). These principles stipulate that delivery of care must be safe, effective, patient centred, timely, efficient, and equitable. We would argue that equitable care is key to overcoming hidden curriculum human factors, such as stigma regarding mental illness that may impede a rational mental health care system or assumption of accountabilities by providers (Knaak et al. 2015; Ungar and Knaak 2013). Implementation of international quality-of-care metrics across mental health services would allow for a better understanding of outcome and recovery. A recent Canadian study found that "mental health high-cost patients", defined as those that acquire $50 \%$ or more of their total healthcare costs within the mental health domain, use $30 \%$ more healthcare expenses per capita compared with non-mental health high-cost patients (de Oliveira et al. 2016). Furthermore, these health costs primarily resulted from psychiatric hospitalizations (de Oliveira et al. 2016). Between 2003 and 2004 , it was found that $37 \%$ of patients with a mental illness were readmitted to an acute care hospital within 1 year of being discharged in Canada as compared to $27.3 \%$ of patients with no mental illness (Canadian Institute for Health Information 2006; Madi et al. 2007). Similarly, within the USA one study cited a $22.3 \%$ rate of readmission for individuals with schizophrenia and other psychotic disorders within 1 year of discharge (Elixhauser and Steiner 2013). Implementation of quality-of-care metrics across psychiatric care internationally has been suggested to aid with these difficulties (Hermann et al. 2006; Fisher et al. 2013). Fisher et al. (2013) describe the frequency of outcome metrics as compared with recovery metrics-possibly due to the dearth of measurable indicators within the latter domain. Nevertheless, understanding what contributes to long-term recovery is essential to improving the mental health system and ensuring individuals achieve periods of wellness for as long as possible. Overall, we believe that the purpose of accountabilities is to improve the system and ensure best practice models of care.

\section{Conclusions}

We believe that the strength and value of the Bauhaus design is in its broad yet simple synthesis of the mental health care system. Ideally, the healthcare system should be designed on principles of rationality, where form follows function, allowing for patient needs to be the foundation for which service and healthcare delivery is structured. Unfortunately, the current mental health system is often not rational and tends to be fragmented and siloed. It often tends to be influenced by non-rational incentives, such as politics, resistance to change, and provider-centred needs. Obstacles to redesigning models of care may also be rooted in history and out-dated philosophies of how systems should be set up. In addition, of course, are the all-too-harsh obstacles of inadequate structural spaces (i.e., inability 
to co-locate) as well as declining resources that could be devoted to creating enablers, such as multidisciplinary teams. Provider-driven needs can sometimes overshadow the needs of patients as redesigning the system might be viewed as "overwhelming" or "staggering". However, often when the flow and process of patient care becomes efficient and needs are matched with resources, the entire system begins to move in a manner that outweighs the potential growing pains of change and restructuring.

Building on a number of other excellent models (Durbin et al. 2001; WHO 2003; Dewa et al. 2010; Government of British Columbia-Ministry of Health 2012), our model has a few differentiating strengths: (1) it highlights and includes the crucial role of primary care providers in the mental health system; (2) it explicitly acknowledges the role of acute ambulatory transitional care as distinct from lower acuity solo practitioner models; (3) it identifies the utility of ambulatory transitional care services within higher acuity provider organizations, such as general hospitals; (4) it acknowledges that both acute care general hospitals and tertiary specialty hospitals are functioning at the highest tier of illness acuity risk and severity; (5) it acknowledges that similar providers can function at different levels of healthcare regardless of skill set; and (6) it recognizes that a successful system requires enablers at all levels-policy, legislation, geographic facilities, skilled providers, e-health technology, and social housing. Currently, enablers are haphazard and poorly understood, and measures of accountabilities are relatively absent in the healthcare system. Both enablers and accountabilities should be integrated into a quality-of-care framework with appropriate rewards linked to acuity, skill, and complexity.

As stated earlier, we recognize that no single model of care will meet all needs. Instead, a continuum of services and models of care is required to address and support the continuum of patient needs organized in the form of a system that serves its function of patient-centred care. We believe that if organizations can begin to make the types of changes discussed in our model, both patients and providers will experience better quality mental health services.

\section{Acknowledgements}

We would like to thank the anonymous reviewers for their helpful feedback which improved our manuscript. We would also like to disclose that there was no funding obtained for this work.

\section{Author contributions}

Conceived and designed the study: TU, MT. Contributed resources: TU, MT, VS. Drafted or revised the manuscript: TU, MT, VS.

\section{Competing interests}

The authors have declared that no competing interests exist.

\section{Data accessibility statement}

All relevant data are within the paper.

\section{References}

American Association of Community Psychiatrists. 2010. Level of care utilization system for psychiatric and addiction services [online]: Available from http://www.communitypsychiatry.org/aacpassets/docs/ publications/clinical_and_administrative_tools_guidelines/LOCUS Instrument 2010.pdf.

Association for Ambulatory Behavioral Healthcare. 2014. 2015 AABH standards and guidelines: partial hospitalization programs and intensive outpatient programs. Association for Ambulatory Behavioral Healthcare, Portsmouth, Virginia, $92 \mathrm{p}$. 
Bakker D, Kazantzis N, Rickwood D, and Rickard N. 2016. Mental health smartphone apps: review and evidence-based recommendations for future developments. Journal of Medical Internet Research: Mental Health, 3: e7. doi:10.2196/mental.4984.

Berwick DM, Nolan TW, and Whittington J. 2008. The triple aim: care, health, and cost. Health Affairs, 27: 759-769. doi:10.1377/hlthaff.27.3.759.

Bullard MJ, Chan T, Brayman C, Warren D, Musgrave E, Unger B, et al. 2014. Revisions to the Canadian Emergency Department Triage and Acuity Scale (CTAS) guidelines. Canadian Journal of Emergency Medicine, 16: 485-489. PMID:25358280. doi:10.1017/S148180350000350X.

Canadian Association of Emergency Physicians (CAEP). 2016. Canadian Triage and Acuity Scale (CTAS)/Prehospital CTAS (Pre-CTAS) [online]: Available from http://caep.ca/resources/ctas.

Canadian Institute for Health Information. 2006. Hospital mental health services in Canada, 2003-2004. Canadian Institute for Health Information, Ottawa, Ontario [online]: Available from https://secure.cihi.ca/free_products/HMHDB_2003-2004_e.pdf.

Canadian Mental Health Association Toronto. 2015. Assertive Community Treatment (ACT) teams [online]: Available from https://toronto.cmha.ca/programs_services/assertivecommunity-treatment-act-teams/.

Carrigan CG, and Lynch DJ. 2003. Managing suicide attempts: guidelines for the primary care physician. Primary Care Companion to the Journal of Clinical Psychiatry, 5: 169-174. PMID:15213779. doi:10.4088/PCC.v05n0405.

Centers for Medicare \& Medicaid Services. 2015. Accountable care organizations (ACO). Centers for Medicare \& Medicaid Services, Baltimore, Maryland [online]: Available from https://www.cms.gov/ Medicare/Medicare-Fee-for-Service-Payment/ACO/index.html?redirect=/Aco/.

Curran V, Ungar T, and Pauzé E. 2006. Strengthening collaboration through interprofessional education: a resource for collaborative mental health care educators. Canadian Collaborative Mental Health Initiative, Mississauga, Ontario.

de Oliveira C, Cheng J, Vigod S, Rehm J, and Kurdyak P. 2016. Patients with high mental health costs incur over 30 percent more costs than other high-cost patients. Health Affairs, 35: 36-43. doi:10.1377/ hlthaff.2015.0278.

Dewa CS, Jacobson N, Durbin J, Lin E, Zipursky RB, and Goering P. 2010. Examining the effects of enhanced funding for specialized community mental health program on contunuity of care. Canadian Journal of Community Mental Health, 29: 23-40. doi:10.7870/ cjcmh-2010-0032.

Droste M. 2006. The Bauhaus. Taschen, Koln, Germany, 256 p.

Durbin J, Cochrane J, Goering P, and Macfarlane D. 2001. Needs-based planning: evaluation of a level-of-care planning model. Journal of Behavioral Health Services and Research, 28: 67-80. PMID:11330000. doi:10.1007/BF02287235.

Elixhauser A, and Steiner C. 2013. Readmissions to U.S. Hospitals by diagnosis, 2010. HCUP Statistical Brief No. 153 [online]: Available from http://www.hcup-us.ahrq.gov/reports/statbriefs/ sb153.pdf. 
Falk K, and Allebeck P. 2002. Implementing assertive community care for patients with schizophrenia. A case study of co-operation and collaboration between mental health care and social services. Scandinavian Journal of Caring Sciences, 16: 280-286. PMID:12191040. doi:10.1046/j.1471-6712. 2002.00081.x.

Fisher CE, Spaeth-Rublee B, and Pincus HA. 2013. Developing mental health-care quality indicators: toward a common framework. International Journal for Quality in Health Care, 25: 75-80. PMID:23175534. doi:10.1093/intqhe/mzs074.

Franx G, Kroon H, Grimshaw J, Drake R, Grol R, and Wensing M. 2008. Organizational change to transfer knowledge and improve quality and outcomes of care for patients with severe mental illness: a systematic overview of reviews. The Canadian Journal of Psychiatry, 53: 294-305. PMID:18551850. doi:10.1177/070674370805300503.

Government of British Columbia-Ministry of Health. 2012. Integrated models of primary care and mental health and substance use care in the community. Government of British ColumbiaMinistry of Health, Victoria, British Columbia, 141 p.

Granello DH, Granello PF, and Lee F. 1999. Measuring treatment outcomes and client satisfaction in a partial hospitalization program. Journal of Behavioral Health Services and Research, 26: 50-63. PMID:10069141. doi:10.1007/BF02287794.

Greenhalgh T. 2009. WHO/WONCA report-integrating mental health in primary care: a global perspective. London Journal of Primary Care, 2: 81-82. doi:10.1080/17571472.2009.11493254.

Grumbach K. 2009. Redesign of the health care delivery system: a Bauhaus "form follows function" approach. Journal of the American Medical Association, 302: 2363-2364. PMID:19952323. doi:10.1001/jama.2009.1772.

Hermann RC, Mattke S, Somekh D, Silfverhielm H, Goldner E, Glover G, et al. 2006. Quality indicators for international benchmarking of mental health care. International Journal for Quality in Health Care, 18(Suppl. 1): 31-38. PMID:16954514. doi:10.1093/intqhc/mzl025.

Hilty DM, Ferrer DC, Parish MB, Johnston B, Callahan EJ, and Yellowlees PM. 2013. The effectiveness of telemental health: a 2013 review. Telemedicine and e-Health, 19: 444-454. PMID:23697504. doi:10.1089/tmj.2013.0075.

Institute of Medicine Committee on Quality of Health Care in America. 2001. Crossing the quality chasm: a new health system for the 21st century. National Academies Press, Washington, DC, $360 \mathrm{p}$.

Kates N, Craven M, Bishop J, Clinton T, Kraftcheck D, LeClair K, et al. 2016. Shared mental health care in Canada [online]: Available from https:/wwwl.cpa-apc.org/Publications/Position_Papers/Shared.asp.

Kates N, Mazowita G, Lemire F, Jayabarathan A, Bland R, Selby P, et al. 2011. The evolution of collaborative mental health care in Canada: a shared vision for the future. The Canadian Journal of Psychiatry, 56(5): 1-10 [online]: Available from http://www.cfpc.ca/uploadedFiles/Directories/Committees_List/ Collaborative\%20mental\%20health\%20care-2011-49-web-FIN-EN.pdf.

Katon W, and Unützer J. 2011. Consultation psychiatry in the medical home and accountable care organizations: achieving the triple aim. General Hospital Psychiatry, 33: 305-310. PMID:21762825. doi:10.1016/j.genhosppsych.2011.05.011. 
Knaak S, Patten S, and Ungar T. 2015. Mental illness stigma as a quality-of-care problem. The Lancet Psychiatry, 2(10): 863-864. PMID:26462211. doi:10.1016/S2215-0366(15)00382-X.

Kuhn E, Weiss BJ, Taylor KL, Hoffman JE, Ramsey KM, Manber R, et al. 2016. CBT-I Coach: a description and clinician perceptions of a mobile app for cognitive behavioral therapy for insomnia. Journal of Clinical Sleep Medicine, 12: 597-606. PMID:26888586. doi:10.5664/jcsm.5700.

Lenz AS, Del Conte G, Lancaster C, Bailey L, and Vanderpool E. 2014. Evaluation of a partial hospitalization program for adolescents. Counseling Outcome Research and Evaluation, 5: 3-16. doi:10.1177/2150137813518063.

Loeb DF, Bayliss EA, Binswanger IA, Candrian C, and Degruy FV. 2012. Primary care physician perceptions on caring for complex patients with medical and mental illness. Journal of General Internal Medicine, 27: 945-952. PMID:22370766. doi:10.1007/s11606-012-2005-9.

Luoma JB, Martin CE, and Pearson JL. 2002. Contact with mental health and primary care providers before suicide: a review of the evidence. American Journal of Psychiatry, 159: 909-916. PMID:12042175. doi:10.1176/appi.ajp.159.6.909.

Lurie S. 2014. Why can't Canada spend more on mental health? Health, 6: 684-690. doi:10.4236/ health.2014.68089.

Madi N, Zhao H, and Li JF. 2007. Hospital readmissions for patients with mental illness in Canada. Healthcare Quarterly, 10: 30-32. PMID:17491564. doi:10.12927/hcq.2007.18818.

Marshall M. 2003. Acute psychiatric day hospitals are not in fashion, but evidence shows that they provide feasible and effective care. British Medical Journal, 327: 116-117. doi:10.1136/ bmj.327.7407.116.

Murray M, Bodenheimer T, Rittenhouse D, and Grumbach K. 2003. Improving timely access to primary care: case studies of the advanced access model. Journal of the American Medical Association, 289: 1042-1046. PMID:12597761. doi:10.1001/jama.289.8.1042.

Nelson G, Aubry T, and Lafrance A. 2007. A review of the literature on the effectiveness of housing and support, Assertive Community Treatment, and intensive case management interventions for persons with mental illness who have been homeless. American Journal of Orthopsychiatry, 77: 350-361. PMID:17696663. doi:10.1037/0002-9432.77.3.350.

Ontario Ministry of Health and Long-Term Care. 2016a. Transforming Ontario's Health Care System: Community Health Links provide coordinated, efficient and effective care to patients with complex needs [online]: Available from http://www.health.gov.on.ca/en/pro/programs/ transformation/community.aspx.

Ontario Ministry of Health and Long-Term Care. 2016b. Family health teams [online]: Available from http://health.gov.on.ca/en/pro/programs/fht/fht_guides.aspx.

Patel V, Chisholm D, Parikh R, Charlson FJ, Degenhardt L, Dua T, et al. 2016. Addressing the burden of mental, neurological, and substance use disorders: key messages from Disease Control Priorities, 3rd edition. The Lancet, 387: 1672-1685. PMID:26454360. doi:10.1016/S0140-6736(15) 00390-6.

Porter ME, and Lee TH. 2013. The strategy that will fix health care. Harvard Business Review, 91: 50-70 [online]: Available from https://hbr.org/2013/10/the-strategy-that-will-fix-health-care. 
Society of Hospital Medicine. 2016. Hospitalists in psychiatric medicine. Society of Hospital Medicine, Philadelphia, Pennsylvania [online]: Available from https://hospitalmedicine.org/Web/ Membership/HFP/Psychiatric_Hospitalists.aspx.

Spitzer RL, Kroenke K, and Williams JB. 1999. Validation and utility of a self-report version of PRIME-MD: the PHQ primary care study. Primary care evaluation of mental disorders. Patient Health Questionnaire. Journal of the American Medical Association, 282: 1737-1744. PMID:10568646. doi:10.1001/jama.282.18.1737.

Thatte S, Makinen JA, Nguyen HN, Hill EM, and Flament MF. 2013. Partial hospitalization for youth with psychiatric disorders: treatment outcomes and 3-month follow-up. Journal of Nervous and Mental Disease, 201: 429-434. PMID:23595097. doi:10.1097/NMD.0b013e31828e1141.

Ungar T, and Knaak S. 2013. The hidden medical logic of mental health stigma. Australia and New Zealand Journal of Psychiatry, 47(7): 611-612. PMID:23405014. doi:10.1177/0004867413476758.

Ungar T, Goldman S, and Marcus M. 2013. Reversed shared care in mental health: bringing primary physical health care to psychiatric patients. Canadian Journal of Community Mental Health, 32: 1-16. doi:10.7870/cjcmh-2013-022.

World Health Organization (WHO). 2003. Mental health policy, planning and service development: integrating systems and services, integrating people. The optimal mix of services: WHO pyramid framework. Department of Mental Health and Substance Abuse, WHO Geneva: The WHO MIND Project: Mental Improvement for Nations Development, Geneva, Switzerland [online]: Available from http:// www.who.int/mental_health/policy/services/2_Optimal\%20Mix\%20of\%20Services_Infosheet.pdf.

Yanos PT, Vreeland B, Minsky S, Fuller RB, and Roe D. 2009. Partial hospitalization: compatible with evidence-based and recovery-oriented treatment? Journal of Psychosocial Nursing and Mental Health Services, 47: 41-47. PMID:19266975. doi:10.3928/02793695-20090201-15.

York Teaching Hospital. 2016. Your diabetes [online]: Available from http://www.yourdiabetes.org. uk/your-diabetes/model-of-care/.

Zhang MW, Ho RC, Cassin SE, Hawa R, and Sockalingam S. 2015. Online and smartphone based cognitive behavioral therapy for bariatric surgery patients: initial pilot study. Technology and Health Care, 23: 737-744. PMID:26409514. doi:10.3233/THC-151026.

Ziguras SJ, and Stuart GW. 2000. A meta-analysis of the effectiveness of mental health case management over 20 years. Psychiatric Services, 51: 1410-1421. PMID:11058189. doi:10.1176/appi. ps.51.11.1410. 